\title{
Design of Digital Circuit Experiment Course Based on FPGA
}

\section{Lei Zhao}

Electronic Experimental Center, Chengdu University of Information Technology, Chengdu, China

Email: zhaolei@cuit.edu.cn

How to cite this paper: Zhao, L. (2021) Design of Digital Circuit Experiment Course Based on FPGA. World Journal of Engineering and Technology, 9, 346-356. https://doi.org/10.4236/wjet.2021.92024

Received: April 6, 2021

Accepted: May 16, 2021

Published: May 19, 2021

Copyright $\odot 2021$ by author(s) and Scientific Research Publishing Inc. This work is licensed under the Creative Commons Attribution International License (CC BY 4.0).

http://creativecommons.org/licenses/by/4.0/

\begin{abstract}
With the development of integrated circuit, the content of digital circuit experiment course is constantly updated. In order to keep up with the development trend of the Times and make students' professional knowledge meet the needs of the industry, the school adopts the FPGA experimental platform to carry out teaching reform from the two aspects of platform and experiment, and carry out reasonable experimental planning to enrich the experimental content. In this paper, the traditional knowledge points of logic algebra, trigger, timer, counter, decoder and digital tube are organically combined, and the digital clock system is designed and realized. The practice shows that the combination of modern design method and traditional digital circuit teaching method can play a good teaching effect. In this way, students can also fully learn, understand and skillfully use the new technology in the experiment, and in the process of building a comprehensive understanding of digital circuits.
\end{abstract}

\section{Keywords}

Digital Circuit, FPGA, Circuit Design, Software Simulation, Digital Clock System

\section{Introduction}

With the development of integrated circuits, the application of digital technology spreads across all walks of life, and digital logic circuits are rapidly changing from the traditional standardized industry of small integrated circuits to largescale programmable devices [1]. Digital logic circuits have developed rapidly in recent years, evolving from traditional small-scale standardized integrated circuits to large-scale programmable devices [2]. The operating frequency of the devices has increased from less than $100 \mathrm{MHz}$ to tens of $\mathrm{GHz}$. The application of 
devices has also changed from simple combination to complex logic [3]. Realization becomes a complex high-speed digital logic control, various high-speed algorithm realizations and special-purpose microprocessors for constructing special functions, etc. High-density, complex logic and extremely high operating frequency have caused profound and revolutionary changes in design methods, design tools, and verification of current digital logic [4] [5] [6].

Digital circuit experiment is based on "Digital Circuit and Logic Design", which is the basic core course of electronic majors [7] [8]. This professional course requires students to be able to master the analysis and design of digital logic circuits and to use them further. The requirements for practice are relatively high [9]. Therefore, in the whole teaching task, after students master the basic circuit knowledge, digital circuit experiment shoulders the task of cultivating professional application ability, so it has an indispensable position in the overall teaching [10] [11].

In recent years, based on the development of modern digital electronic technology and the educational concept of advancing with the times, the experimental teaching of digital circuits has innovated the experimental platform, experimental content and experimental form [12]. First of all, the traditional experimental method is to build a digital circuit by 74 series gate circuits [13]. Because this method is too far from the realization of the digital circuit industry, our digital circuit experiment platform has transitioned to use CPLD, FPGA and other programmable logic devices [14] [15]. The new platform uses a combination of software and hardware, which can be easily built on any computer, and the experimental equipment is small and portable. Secondly, the experimental content has changed from verification to comprehensive and innovative design experiments, from a single, independent basic circuit realization to a complex system circuit design [16]. As the number of experimental steps increases, possible problems become more complicated [17]. Finally, there are certain changes in the experimental format. Through pre-class preview, program design, sub-module implementation, system integration and other links, the classroom efficiency has been improved, and students' thinking and practical skills have been enhanced [18] [19]. The experimental form has changed from a pure classroom experiment to a form of extracurricular debugging combined with in-class centralized problem solving [20]. These improvement methods aim to promote teaching and learning, making the course of digital circuit conform to the teaching concept of CDIO, and cultivate talents of electronic technology under the background of new engineering education.

\section{Experimental Ideas}

Since the establishment of the digital circuit experiment, some confirmatory experiments on 74 series chips have been the main focus. With the changes in the use, carrier, and design verification methods of modern digital logic circuits, many colleges and universities have reformed digital circuit experiments in terms of experimental content design, device use, design methods and verifica- 
tion methods. After investigation, they found that they can be summarized as follows:

1) Replace the main experimental devices, and replace the original 74 series gate circuits with programmable logic devices such as CPLD and FPGA.

2) Change the experimental form, abandon the experimental form of manually connecting the circuit on the breadboard or experiment box, and replace it with EDA tools to design and debug the circuit.

3) Expand experimental content and introduce comprehensive and innovative design experimental content.

Traditional experimental teaching is based on 74 series chips, which are used to build circuits on the experiment box for experimental research. This method allows students to perform manual wiring, which is more cumbersome, high in time and costly, and out of touch with the industry. After changes, the current digital circuit experiments are basically in line with the current mainstream technology in terms of experimental devices, digital circuit design and verification, and use EDA simulation software for teaching [21]. But this is not to say that there are no problems. In this teaching method, students in the lower grades must not only learn the knowledge of circuits, but also learn the operation of the software. EDA software is powerful, but it is more difficult for students in lower grades to learn, which affects their interest in learning. The digital and electrical experiment belongs to the basic experiment in the electronic professional teaching and training system. Its task is to support theoretical teaching first. There are a lot of content in theoretical teaching about various simple gate circuits and triggers. For this part of the content, how to simple, Intuitive experimental demonstration and verification of them, so as to impress the students, this is a big problem. Some students emphasize circuit design and software simulation, and even think that digital circuit design is equivalent to software simulation, ignoring the importance of verification with experiment boards, and a deviation in the overall understanding of digital circuits. Finally, the connection between comprehensive and innovative design experiment content and basic experiment, proportional distribution, difficulty control and other issues need to be further studied [22].

\section{Digital Circuit Experiment Design Based on FPGA}

Aiming at the problem of students ignoring the experiment board, FPGA is used as the experimental hardware platform. Students are required to download the designed circuit to the board every time they experiment, debug and verify the circuit on the experiment board, and train students to use instruments, software and hardware to learn the capabilities of digital circuits. The difficulty of the experiment is progressive, divided into three levels: basic, expansion and synthesis, to solve the problem of connection between different types of experiments, and finally achieve the goal of designing a complex digital system. Through the teacher's explanation and the students' personal operation, the students will gradually become familiar with the EDA software [23]. The initial experiments 
are relatively simple and basic, with more verifying content and less design content. Simple and intuitive experiments are mainly used to verify the knowledge points of theoretical teaching. Through this part of the experiment, students will become familiar with the operation of the software as soon as possible and prepare for subsequent experiments. This allows students to focus more on circuit design in the design experiment, reducing the problems caused by unskilled operations. Subsequent experiments introduced complex content step by step. Try to minimize the impact of platform and design difficulty changes on students, so that they can gradually master digital circuit development skills.

\subsection{Experimental Platform Reform}

The designed FPGA experimental platform has relatively complete functions, especially in terms of lower board verification and hardware debugging, which shows good operability and flexibility. Figure 1 is a simplified block diagram of the experimental hardware platform module. According to the experimental application, it can be summarized as: programmable logic device, FPGA uses ALERA's CYCLONE V series, containing $85 \mathrm{~K}$ programmable logic device, 4450 $\mathrm{Kb}$ embedded memory unit; on-board resources, Peripherals are equipped with buttons, LEDs, digital tubes and other devices for off-board verification. 72 GPIOs are reserved on the board as expansion ports for supply expansion, function customization and debugging; supporting components, providing external clocks, USB-Blaster debuggers, etc. for experiments support.

The Quartus II 13.1 used in the development environment includes solutions for all stages of the FPGA development process. It has the advantages of short design cycle, easy operation, strong design flexibility, and rich debugging methods. Based on the above platform, the circuit design and verification methods are changed, so that students are well trained in both soft and hard aspects. Students first complete design input and synthesis on Quartus II. The specific adaptation chip and placement and routing are automatically completed by the computer, replacing the traditional manual routing experiment. Then use Quartus II to call ModelSim-Altera 10.1d co-simulation and debugging, and test

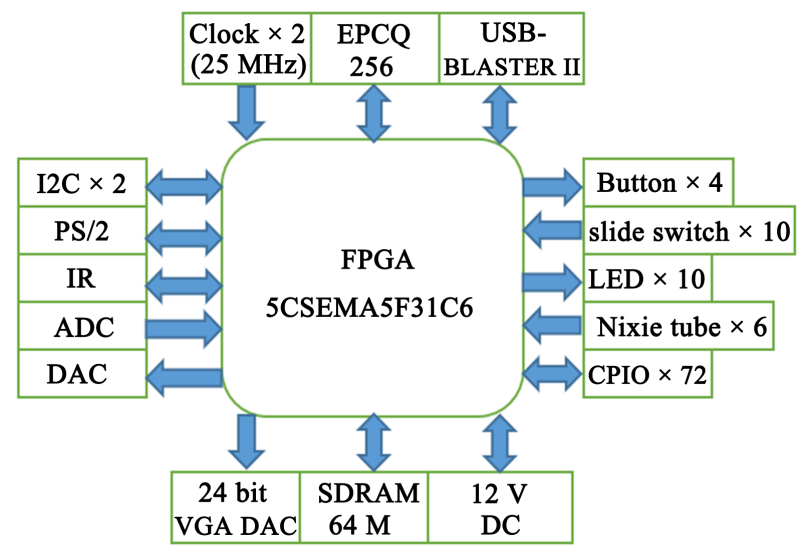

Figure 1. The diagram of FPGA experimental system. 
the circuit function preliminarily without the aid of instruments. When verifying on the board, download the generated sof file to the actual operation of the FPGA observation circuit. The experiment effect can be designed according to the onboard resources of the experiment platform, which adds to the fun of the experiment. For comprehensive debugging, the experimental platform reserves debugging ports and FPGA resources to support various signal observation methods such as instrument testing and online logic analyzer monitoring.

The new platform eliminates the need for manual wiring on the experimental box, greatly reducing unnecessary labor time and allowing students to focus on circuit design while significantly improving efficiency. For example, the overall experiment time was reduced by $20 \%$ for the LED flow-light experiment after the new platform was adopted. For the traffic light design, the experiment time was reduced by $15 \%$.

\subsection{Experimental Planning and Content Reform}

The selection of experiment content not only considers the theoretical and holistic nature of the course itself, but also considers the systematic nature of the experiment and subsequent professional courses. The experimental system structure is shown in Figure 2. The experiment covers three stages to master a new technology, and a complete experimental system is formed by several experimental items with increasing difficulty. The preliminary experiment is the basis of the subsequent experiment, and the subsequent experiment is the application of the preliminary experiment.

The introductory experiment is for beginners to familiarize themselves with the new experimental platform and to intuitively verify the theoretical teaching

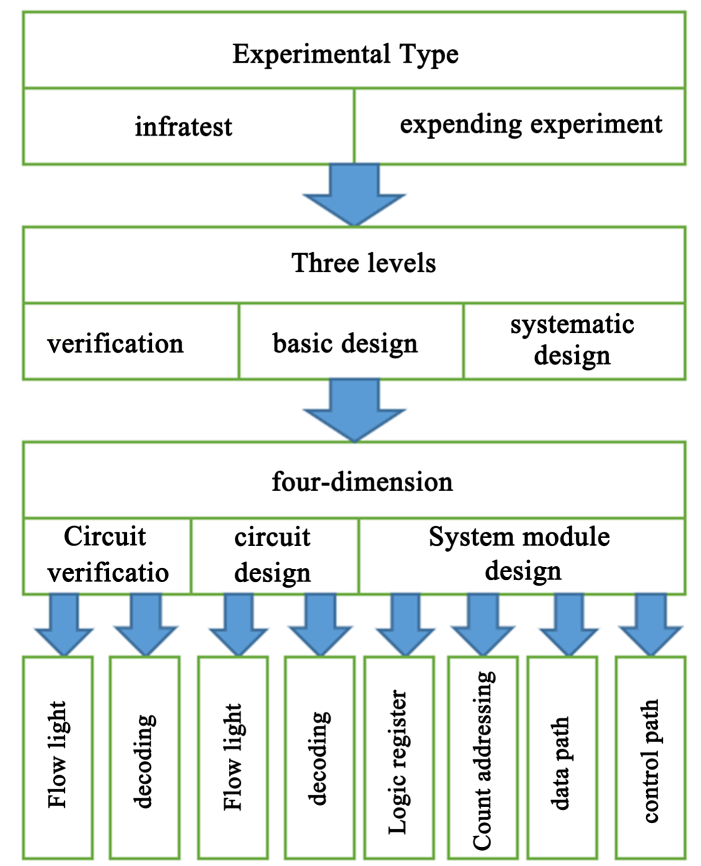

Figure 2. Schematic diagram of digital circuit experimental structure. 
knowledge points based on the platform. This experiment calls the common device library that comes with quartus, and uses the method of schematic drawing to simulate traditional experiments to verify various simple gate circuits, flip-flops and module circuits on a breadboard or experiment box. The experiment requires students to verify the functions of the 74LS138 and the 4-input 16-output decoder composed of 2 pieces of 74LS138 through the lower board and the instrument on the FPGA platform. The operation involved in the whole process is very basic, which can avoid the problem of poor reliability and flexibility of manual wiring.

The basic design part self-modeling to realize the 74LS138 in the verification circuit is used to compare and analyze the difference between the standardized module circuit and the self-modeling design circuit. The transition from the traditional experimental form to the self-designed circuit using verilog HDL. Each experimental project at this stage requires a complete experimental process covering hardware modeling, software simulation, board verification, and comprehensive debugging. In the basic circuit design, the concept of timing is added to the water lamp circuit, and the circuit complexity is improved compared with the decoder. The advanced part of the basic design designs and verifies the simplified instruction set CPU functional components, such as addressing units, arithmetic logic units, etc., obtained from the combination of basic circuits or expanded functions. The circuit structure designed in the early stage of the experiment is simple, and students can devote more energy to the study of EDA software. With the gradual increase in modeling complexity, the software operation becomes more and more proficient, and more attention can be paid to the learning of logic description language and the improvement of circuit analysis and debugging capabilities in the later period.

The expansion experiment begins with the practical sequence of module circuit, subsystem circuit to system circuit design, and gradually integrates the basic circuit into components, and then the components work together to form a simplified CPU system. According to the pre-set instruction set architecture, bus structure, circuit structure and other index parameters, the students design the number of instructions to be 24 . The address, data bus, and I/O interface are all 16-bit simplified instruction set CPUs. The lower board verification link requires the CPU to automatically read the instructions in the memory and operate the I/O interface level to realize the running light. Students design circuits to implement CPU functions such as data access, data processing, and interface control, which will help students to deeply understand the supporting role of digital logic circuits for subsequent courses such as microcomputer principles, computer composition principles, and ASIC design.

\section{Module Realization of the Experimental System}

\subsection{Key Module}

In order for the system to accurately identify the operation of the buttons, it is 
necessary to perform debounce and release detection processing on the keys. Key debounce can be divided into hardware debounce and software debounce. In order to simplify the experimental process, this article uses software debounce to obtain stability. The theoretical button jitter time is $5-10 \mathrm{~ms}$. For this, the button module first defines a timer with a duration of $15 \mathrm{~ms}$, and then judges the level signal of the corresponding port after the button is pressed for $15 \mathrm{~ms}$.

The realization method of letting go detection is shown in Formula (1):

$$
\text { key_press = key_reg \& ( key_reg_next), }
$$

In the Formula (1), key_press is a register variable indicating whether the key press is valid, key_reg stores the state of the key at the current moment, and key_reg_next is the state of the key at the next moment. From Formula (1), it can be seen that if key_press is 1, key_reg must be 1 and key_reg_next It must be 0 , and its corresponding meaning is: only the key is not pressed at the current moment, and is pressed at the next moment, it is recognized as a valid key trigger signal. Figure 3 is the gate-level circuit diagram of the release detection method.

\subsection{Timing Module}

The timing module first defines a timer with a duration of $1 \mathrm{~s}$ as the counter clock reference. The design idea of this module is to use the 1s timer to complete the counting of unit seconds, and then according to the unit second and unit minute and unit minute and unit hour. Figure 3 is a block diagram of the realization of the unit second. The second digit is composed of the second digit and the second tens digit, and the value is cyclically changed between 0 and 59 . The realization process of the unit second is the modulo sixty counter. Implementation process. The module first defines the modulus ten counter to complete the counting of the second digit, and then defines the modulus six counter, and cascades the modulus ten counter and the modulus six counter in a serial carry way to complete the realization of the modulus sixty counter. Each one After the $60 \mathrm{~s}$ count is completed, the units digit and tens digit of the second are cleared, and the carry output bit of the tens digit of the second is carried to the units digit of the unit minute to complete the clock halving.

\subsection{Digital Tube Module}

The six eight-segment digital tubes of the experimental platform are connected

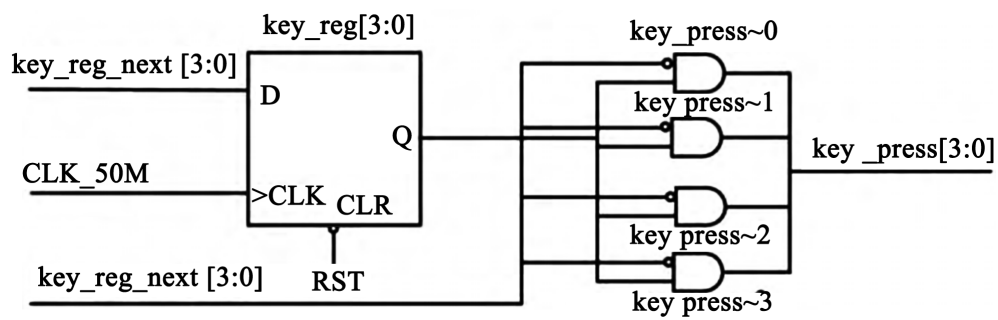

Figure 3. Gate level circuit diagram of detection function realization. 
to the SEG_EN0 - SEG_EN5 pins in the FPGA as the chip selection terminal of the FPGA using a common cathode method. The a - $g$ and decimal point dp pins of the 6 digital tubes are connected in parallel to the FPGA's SEG_DATAa SEG_DATADP pins are used as data input terminals. The experiment uses dynamic scanning to allow 6 digital tubes to display the current hour, minute, and second information at the same time. The module defines a $1 \mathrm{~ms}$ timer as the scanning period of the dynamic scanning digital tube. In the period of time, the time information to be displayed is decoded first. According to the actual situation, only the 10 numbers 0 - 9 are decoded. After the decoding is completed, the decoded result is assigned to SEG_DATAx $(x=a, b . . . D P)$. At the same time, pull down the corresponding digital tube chip selection end, and the corresponding time information will be displayed on the digital tube. The next time information will be scanned and displayed every $1 \mathrm{~ms}$. Visual persistence, when you see the hour, minute, and second information, it will be displayed on the digital tube at the same time.

\subsection{Top-Level Module}

After using the hardware description language (Verilog HDL) to describe the functions to be implemented by each module, write the top-level module to connect the sub-modules. Use the analysis and synthesis tools of Quartus II to compile the project and generate the RTL (Register Transfer Level) function. It can be seen from the figure that the digital clock system is divided into 4 modules, and the internal ports and external ports of the system are consistent with the system to be designed.

\subsection{Hardware Verification}

After using Model to perform software simulation on each module and the entire circuit, ensure that the functions of each module are normal, and finally download the compiled "sof" file to the FPGA development board through the download line. First, complete the clock hour, minute and second setting by pressing the button. Then press the start button to start timing. When the clock reaches the hour, the buzzer can beep for 1s. After actual inspection, the designed digital clock can operate normally and meet the expected design requirements.

\section{Summary and Conclusions}

This article takes the current problems of digital circuit experiments as a starting point, and proposes practical reform measures from both the experimental platform and experimental content. The reformed digital circuit basic experiment can not only simultaneously support the "digital logic circuit and system" theoretical course, but also provide a complete set of modern digital integrated circuit development tutorials. Through experimental design from easy to difficult, students construct a correct understanding of modern digital circuit design in 
experimental teaching, train digital circuit design, analysis, verification and debugging capabilities, and effectively cultivate students' comprehensive quality of engineering practice. Since the circuit design method and verification method of each experimental project are basically the same, the difficulty of the experiment is reflected in the difference in modeling complexity. This difference depends on the level of the CPU. The pre-order experiment is the basis of the post-order experiment, and the post-order experiment is the application of the pre-order experiment. The students' logic description language application ability and circuit analysis and debugging ability gradually increase as the difficulty of the experiment increases. Get trained.

The experimental reform has been applied for two years, and has been continuously adjusted through teaching practice and student feedback, and has achieved remarkable results. Practice has proved that the experiment can not only help students better learn and understand theoretical knowledge, but also enable them to master the method of designing and implementing digital systems based on FPGA. According to the questionnaire survey, $90 \%$ of the students think that the experiments conducted on the new platform can stimulate their enthusiasm for learning and have the desire for further research. This percentage is higher than the results of the questionnaire when using the old platform. In the return visit, most students said that the experiment was very helpful to their understanding of the basic concepts and principles in the "Digital circuit" course.

\section{Acknowledgements}

This work was funded by Collaboration between industry and school Project of Ministry of Education: "Innovative teaching and training of PAEE system in electronic information major” (No. 201901103032).

\section{Conflicts of Interest}

The author declares no conflicts of interest regarding the publication of this paper.

\section{References}

[1] Carroll, L., Lee, J.S., Scarcella, C., et al. (2016) Photonic Packaging: Transforming Silicon Photonic Integrated Circuits into Photonic Devices. Applied Sciences, 6, 426. https://doi.org/10.3390/app6120426

[2] Grout, I.A. (2005) Integrated Circuit Test Engineering: Modern Techniques. Springer Science \& Business Media, Berlin.

[3] Ayers, J.E. (2018) Digital Integrated Circuits: Analysis and Design. CRC Press, Boca Raton. https://doi.org/10.1201/b12491

[4] Kocur, M., Kozak, S. and Dvorscak, B. (2014) Design and Implementation of FPGADigital Based PID Controller. Proceedings of the 2014 15th International Carpathian Control Conference (ICCC), Velke Karlovice, 28-30 May 2014, 233-236. https://doi.org/10.1109/CarpathianCC.2014.6843603 
[5] Sankarayya, N., Roy, K. and Bhattacharya, D. (1997) Algorithms for Low Power and High Speed FIR Filter Realization Using Differential Coefficients. IEEE Transactions on Circuits and Systems II: Analog and Digital Signal Processing, 44, 488-497. https://doi.org/10.1109/82.592582

[6] Noll, T.G. (1991) Carry-Save Architectures for High-Speed Digital Signal Processing. Journal of VLSI Signal Processing Systems for Signal, Image and Video Technology, 3, 121-140. https://doi.org/10.1007/BF00927839

[7] Martin, K.W. (2000) Digital Integrated Circuit Design. Oxford University Press, New York.

[8] Holdsworth, B. and Woods, C. (2002) Digital Logic Design. Elsevier, Amsterdam.

[9] Zemva, A., Trost, A. and Zajc, B. (1998) A Rapid Prototyping Environment for Teaching Digital Logic Design. IEEE Transactions on Education, 41, 342.

https://doi.org/10.1109/13.728275

[10] Obaid, Z.A., Sulaiman, N. and Hamidon, M.N. (2009) FPGA-Based Implementation of Digital Logic Design Using Altera DE2 Board. International Journal of Computer Science and Network Security, 9, 186-194.

[11] Farooq, U., Marrakchi, Z. and Mehrez, H. (2012) FPGA Architectures: An Overview. In: Tree-Based Heterogeneous FPGA Architectures, Springer, New York, 7-48.

https://doi.org/10.1007/978-1-4614-3594-5 2

[12] Sulaiman, N., Obaid, Z.A., Marhaban, M.H., et al. (2009) FPGA-Based Fuzzy Logic: Design and Applications-A Review. International Journal of Engineering and Technology, 1, 491. https://doi.org/10.7763/IJET.2009.V1.90

[13] Lala, P.K. and Burress, A.L. (2003) Self-Checking Logic Design for FPGA Implementation. IEEE Transactions on Instrumentation and Measurement, 52, 13911398. https://doi.org/10.1109/TIM.2003.818545

[14] Shi, J. and Deng, C. (2010) Digital Circuit Experiment System Based on FPGA. 2010 International Conference on E-Health Networking Digital Ecosystems and Technologies (EDT), Vol. 2, 277-280. https://doi.org/10.1109/EDT.2010.5496371

[15] Brown, S. and Rose, J. (1996) Architecture of FPGAs and CPLDs: A Tutorial. IEEE Design and Test of Computers, 13, 42-57. https://doi.org/10.1109/54.500200

[16] Tzou, Y.Y. and Hsu, H.J. (1997) FPGA Realization of Space-Vector PWM Control IC for Three-Phase PWM Inverters. IEEE Transactions on Power Electronics, 12, 953-963. https://doi.org/10.1109/63.641493

[17] Tuna, M. and Fidan, C.B. (2016) Electronic Circuit Design, Implementation and FPGA-Based Realization of a New 3D Chaotic System with Single Equilibrium Point. Optik, 127, 11786-11799. https://doi.org/10.1016/j.ijleo.2016.09.087

[18] Jung, S.L., Chang, M.Y., Jyang, J.Y., et al. (1999) Design and Implementation of an FPGA-Based Control IC for AC-Voltage Regulation. IEEE Transactions on Power Electronics, 14, 522-532. https://doi.org/10.1109/63.761696

[19] Vaidyanathan, S., Pehlivan, I., Dolvis, L.G., et al. (2020) A Novel ANN-Based Four-Dimensional Two-Disk Hyperchaotic Dynamical System, Bifurcation Analysis, Circuit Realisation and FPGA-Based TRNG Implementation. International Journal of Computer Applications in Technology, 62, 20-35. https://doi.org/10.1504/IJCAT.2020.103921

[20] Sambas, A., Vaidyanathan, S., Tlelo-Cuautle, E., et al. (2019) A Novel Chaotic System with Two Circles of Equilibrium Points: Multistability, Electronic Circuit and FPGA Realization. Electronics, 8, 1211. https://doi.org/10.3390/electronics8111211

[21] Qin, L., Wang, X. and Guan, J. (2009) Application of EDA Simulation in Designing Experiments of Computer Organization. Research and Exploration in Laboratory, 
28, 79-82.

[22] Tian, J. and Xia, L. (2005) Teaching Practice of EDA-Based Electronic Technology. Theory and Practice of Education, 12, 22.

[23] Jie, D. (2012) Simulation Software Applied in Electronic Technology Teaching. 2012 IEEE International Conference on Industrial Control and Electronics Engineering, Xi'an, 23-25 August 2012, 925-927. https://doi.org/10.1109/ICICEE.2012.245 